\title{
O suporte social como fator de proteção para as mães de crianças com Síndrome da Zika Congênita
}

\author{
Social support as a protective factor for the mothers of children \\ with Congenital Zika Syndrome
}

Tiago Jessé Souza de Lima (https://orcid.org/0000-0001-8840-4285) ${ }^{1}$

Luana Elayne Cunha de Souza (https://orcid.org/0000-0001-9425-9598) ${ }^{2}$

${ }^{1}$ Instituto de Psicologia, Universidade de Brasília. Campus da UnB, Área Universitária. 73345-010 Brasília DF Brasil. tiago. souzalima@hotmail.com

${ }^{2}$ Programa de Pós-

Graduação em Psicologia,

Universidade de Fortaleza.

Fortaleza CE Brasil.

\begin{abstract}
Mothers of children with CZS face exceptional challenges caring for their offspring. Due to this reality, the availability of social support can function as an important protective factor. In this respect, this study aimed to evaluate the role of informal and formal social support in mental health, parental self-efficacy and satisfaction in the life of mothers of children with CZS. A total of $69 \mathrm{mo-}$ thers of children with CZS ( $M=26.4$ years; $S D=$ 6.23) living in the state of Ceará, Brazil participated in this study. They filled out the Social Support Satisfaction Scale, General Health Questionnaire, Parental Self-efficacy Scale and Satisfaction with Life Scale. The results show that the greater perception of informal social support significantly enables better mental health and satisfaction with life levels. In addition, access to formal social support significantly enables enhanced perception of parental self-efficacy. The results observed with the mothers of children with CZS are consistent with those reported in other studies with mothers of children with chronic conditions, pointing out that formal and informal social support have different effects on mental health, parental self-efficacy, and satisfaction with life, even controlling the effect of sociodemographic variables.

Key words Social support, Mental health, Zika virus, Self-efficacy, Personal satisfaction
\end{abstract}

Resumo As mães de crianças com Sindrome da Zika Congênita (SZC) enfrentam desafios excepcionais no cuidado com seus filhos. Mediante essa realidade, a disponibilidade de suporte social pode funcionar como um importante fator de proteção. Nesse sentido, esse trabalho teve por objetivo avaliar o papel do suporte social informal e formal na saúde mental, autoeficácia parental e satisfação com a vida de mães de crianças com SZC. Participaram desse estudo 69 mães de crianças com SZC $(M=26,4$ anos; $D P=6,23)$, residentes no estado do Ceará, Brasil. Elas responderam a Escala de Satisfação com o Suporte Social, Questionário de Saúde Geral, Escala de Autoeficácia Parental e Escala de Satisfação com a Vida. Os resultados apontam que a maior percepção de suporte social informal prediz significativamente melhores niveis de saúde mental e satisfação com a vida. Ademais, o acesso ao suporte social formal prediz significativamente uma maior percepção de autoeficácia parental. Os resultados observados com as mães de crianças com SZC são coerentes com os relatados em outros estudos, apontando que o suporte social formal e informal apresentam efeitos distintos na saúde mental, autoeficácia parental e satisfação com a vida, cabendo observar que esses efeitos ocorreram mesmo controlando o efeito de variáveis sociodemográficas.

Palavras-chave Suporte social, Saúde mental, Zika vírus, Autoeficácia, Satisfação pessoal 


\section{Introdução}

As mães de crianças com condições crônicas, portadoras da Síndrome da Zika Congênita (SZC), transtorno do espectro autista e Síndrome de Down, por exemplo, enfrentam desafios excepcionais no cuidado com seus filhos, já que eles exigem um maior envolvimento dos pais, por terem múltiplas necessidades de cuidado que vão além das de uma criança da mesma idade com desenvolvimento típico ${ }^{1-3}$. De acordo com Souza et al. ${ }^{3}$, as mães e pais dessas crianças precisam lidar com a aceitação das condições e limitações dos filhos. Neste sentido, os pais procuram se ajustar e gerenciar as dificuldades dos filhos, podendo enfrentar sofrimento emocional significativo, conflitos no relacionamento, problemas financeiros, estigmatização, isolamento social e atitudes sociais negativas, além do tempo consumido nas consultas a diversos especialistas.

Não é de surpreender que mães de crianças com condições crônicas relatem consistentemente maior estresse percebido, maior carga de cuidado e mais sintomas depressivos do que mães de crianças com desenvolvimento típico ${ }^{1}$. Em relação às mães de crianças com SCZ, alguns estudos empíricos ${ }^{3-6}$ apontam um quadro similar ao observado com mães de crianças com outras condições crônicas, apontando níveis elevados de ansiedade, fadiga, menor qualidade de vida e pior saúde mental de forma global. Ademais, o papel social de cuidar dos filhos é naturalizado pela sociedade como uma demanda quase exclusiva da mulher, o que pode acarretar mais consequências negativas para a saúde mental dessas mães. No entanto, os resultados negativos para as mães não podem ser simplesmente explicados pelos efeitos de criar um filho com condição crônica em si, mas também pelas características dessa condição crônica ${ }^{7}$. A este respeito, as famílias de crianças com SZC podem estar expostas a condições ainda mais críticas, sobretudo devido às incertezas das consequências da SZC, ainda pouco estudadas na literatura médica, para a vida futura da criança ${ }^{8}$.

Todavia, diversos estudos na literatura têm se dedicado a identificar os fatores associados com a variação nos níveis de saúde mental de mães de crianças com condições crônicas. Alguns estudos apontam que a variação na saúde mental das mães está associada às características da condição crônica em si, como dito anteriormente, a exemplo da severidade do comprometimento motor da criança ${ }^{9}$, as características psicológicas dos pais, a percepção de autoeficácia ${ }^{10}$ e estratégias de coping ${ }^{11}$, e aos fatores contextuais, como o suporte social provido por familiares, amigos e por profissionais dos serviços de saúde ${ }^{1,12}$.

Nesse contexto, a disponibilidade de suporte social pode funcionar como um importante fator de proteção para a saúde mental dessas mães. O suporte social é definido como a prestação de assistência emocional, informativa e instrumental que um indivíduo recebe a partir de pessoas significativas nas suas redes sociais ${ }^{13}$. O suporte emocional refere-se a demonstrações de amor e carinho, estima e valor, encorajamento e simpatia; o suporte informativo diz respeito à provisão de orientações e aconselhamento que podem ajudar a pessoa resolver problemas e sobre possíveis cursos de ação que devem adotar; já o suporte instrumental consiste em oferecer ou fornecer assistência comportamental ou material com tarefas ou problemas práticos ${ }^{13}$.

$\mathrm{O}$ acesso das mães de crianças com condições crônicas a essas funções do suporte social depende das relações sociais significativas mantidas com pessoas próximas, ou seja, do capital social, que irá condicionar o suporte de caráter emocional $^{13}$; assim como de fatores sociais estruturais, como a comunidade, as instituições e a cultura na qual estão inseridas ${ }^{14}$. Essa estrutura social tem importante papel no desenvolvimento de crianças com condições crônicas, já que influenciam o acesso a serviços de apoio formal ${ }^{15}$, a exemplo dos ofertados pelas políticas públicas voltadas à saúde da população, afetando a disponibilidade de suporte informativo e instrumental ${ }^{1,13}$.

Dessa forma, o suporte social pode ser proveniente de fontes informais e formais. O suporte informal é fornecido por alguém na rede social do indivíduo e geralmente é oferecido voluntariamente - na maioria das vezes por familiares, amigos, vizinhos ou conhecidos - mais vinculado ao apoio emocional ${ }^{16}$. O suporte social formal refere-se ao suporte prestado por uma organização ou por profissionais, de forma pública ou privada. Nessa categoria, podem ser incluídos os profissionais da saúde (médicos, psicólogos, fisioterapeutas, entre outros), que provêm cuidados, informações e orientação profissional e recursos práticos para ajudar na gestão da condição crônica, primariamente vinculados ao apoio informativo e instrumental ${ }^{16}$, embora possam também ser fonte secundária de apoio emocional.

Estudos realizados com pais e mães de crianças com condições crônicas têm apontado que a presença de suporte social está associada a níveis mais baixos de depressão e estresse ${ }^{17,18}$, a menor sobrecarga de cuidado percebida ${ }^{19}$ e a níveis mais elevados de autoestima e satisfação com a vida ${ }^{20}$. 
Em relação às mães de crianças com SZC, não foi encontrado nenhum estudo empírico na literatura que tivesse por objetivo avaliar o efeito do suporte social na saúde mental dessas mães (busca conduzida no Google Acadêmico em sete de fevereiro de 2020, com as palavras-chave "suporte social", "zika" e "saúde mental”), embora esse seja um tema enfatizado em guias para a prática profissional com mães de crianças com SZC. Nesse sentido, esse trabalho teve por objetivo avaliar o papel preditivo do suporte social informal e formal na saúde mental, autoeficácia materna e satisfação com a vida de mães de crianças com SZC.

Especificamente, propomos que a maior percepção de suporte social informal irá predizer significativamente melhores níveis de saúde mental (hipótese 1) e de satisfação com a vida (hipótese 2) de mães de crianças com SZC. De fato, o suporte social informal desempenha um papel importante, na ajuda dos cuidadores a reduzir os sentimentos de isolamento e desamparo geralmente associados à criação de um filho com condição crônica ${ }^{21}$, na melhora da satisfação com a vida ${ }^{20}$ e, consequentemente, na promoção da sua saúde mental ${ }^{17}$. Em relação ao suporte formal, propomos que o maior acesso a esse suporte irá predizer significativamente maiores níveis de autoeficácia parental (hipótese 3) e, consequentemente, maiores níveis de saúde mental (hipótese 4). O suporte social formal pode desempenhar um importante papel na predição da autoeficácia materna, ou seja, nas crenças que as mães têm sobre suas habilidades de prover cuidados aos filhos de forma bem-sucedida, já que o acesso a serviços de saúde constituem uma fonte mais propícia de apoio informativo e instrumental ${ }^{1,16}$. O suporte social formal, ao impactar positivamente na autoeficácia parental, pode também contribuir para melhores níveis de saúde mental, já que alguns estudos apontam que níveis mais elevados de autoeficácia parental estão associados a menores níveis de depressão e estresse ${ }^{22,23}$.

\section{Método}

\section{Participantes}

Participaram desse estudo 69 mães de crianças com SZC, residentes no estado do Ceará, com média de idade de 26,4 anos $(D P=6,23)$, variando entre 16 e 42 anos. Em relação à cor da pele, a maioria das mães se declararam parda $(62,3 \%)$ e branca (22\%). 33,9\% das mães indicaram ter Ensino Fundamental completo ou incompleto,
51,5\% indicaram ter Ensino Médio completo ou incompleto e 14,7\% indicaram ter Ensino Superior completo ou incompleto. A maioria das mães estavam desempregadas ( $81 \%$ ) no momento da pesquisa, e $67,6 \%$ indicaram que trabalhavam antes de ter o filho com SZC e agora apenas $13,4 \%$ declararam trabalhar. A renda familiar mensal média é de 1,3 salários mínimos ( $\mathrm{DP}=$ 1,07). Quanto à situação conjugal, a maioria das mães indicou estar casada ou em união-estável com o parceiro $(69,6 \%)$, em que $42 \%$ indicou não ter outro filho e $56,5 \%$ indicou ter ao menos mais um filho, além da criança com SZC. Em relação ao acesso aos serviços de saúde, 52\% indicou usar apenas os serviços públicos, enquanto $34,8 \%$ indicaram possuir também acesso a planos de saúde privados.

\section{Instrumentos}

A percepção de suporte social informal foi mensurada através da Escala de Satisfação com o Suporte Social (ESSS) ${ }^{24}$, composta por 15 afirmações que medem quatro dimensões: Satisfação com as amizades, Intimidade, Satisfação com a família e Atividades sociais. Os participantes indicaram o quanto concordavam com cada item (por exemplo: estou satisfeito com a quantidade de tempo que passo com os meus amigos; por vezes sinto-me só no mundo e sem apoio) através de uma escala de cinco pontos, variando entre 1 (discordo muito) e 5 (concordo muito). A ESSS apresentou um alfa de Cronbach de 0,83 .

Para avaliar o suporte formal foi solicitado que as mães indicassem se elas e seu filhos eram acompanhadas por profissionais das políticas públicas de atenção à saúde $(0=$ não; $1=\operatorname{sim})$, especificando quais posteriormente (assistente social, agente de saúde, dentista, enfermeiro, fisioterapeuta, fonoaudiólogo, médico, nutricionista, psicólogo, terapeuta ocupacional). Para o cálculo da variável suporte social formal foi realizado um somatório do número de profissionais, indicado pela mãe, que provêm assistência direta ao filho.

A saúde mental foi mensurada através do Questionário de Saúde Geral (QSG-12) ${ }^{25}$, composto por 12 itens que medem sintomas psiquiátricos não psicóticos. No caso de itens negativos, as alternativas de resposta variam de $1=$ absolutamente não, a 4 = muito mais que de costume; em caso de itens positivos as respostas variaram de $1=$ mais que de costume, a $4=$ muito menos que de costume. Maiores pontuações são indicativas de pior nível de saúde mental. $\mathrm{O}$ índice de 
consistência interna da escala também foi adequado $(\alpha=0,80)$.

A autoeficácia parental foi mensurada através de oito itens da Revised Caregiving Appraisal Scales, subescala Caregiving mastery ${ }^{26}$ adaptados para a realidade de cuidados das mães de crianças com SZC. As mães eram solicitadas a indicarem sua concordância com afirmações relacionadas aos cuidados dos filhos com SZC, utilizando uma escala de resposta variando de 1 (discordo muito) a 5 (concordo muito). Essa escala apresentou um alfa de Cronbach de 0,79.

Para avaliar a satisfação com a vida foi utilizada a Escala de Satisfação com a Vida (Satisfaction with Life Scale - SWLS) ${ }^{27}$. A SWLS é composta por cinco itens que avaliam a satisfação com aspectos gerais da vida. As respostas são dadas de acordo com uma escala de 7 pontos $(1=$ discordo totalmente a $7=$ concordo totalmente), tendo apresentado valor de alfa de Cronbach igual a 0,72.

Por fim, os participantes responderam um questionário sociodemográfico, contendo perguntas sobre idade, situação conjugal, número de filhos, escolaridade, renda, uso de serviços públicos e privados de saúde, entre outras.

\section{Procedimento}

A coleta de dados foi realizada durante um mutirão de atendimento multiprofissional a crianças diagnosticados com SZC realizado no estado do Ceará. As mães eram abordadas individualmente por seis pesquisadoras devidamente treinadas para essa tarefa. Nesse momento inicial o objetivo da pesquisa era explicado, assim como era salientado o caráter voluntário e anônimo das respostas. Aquelas que concordaram em participar da pesquisa assinaram o termo de consentimento livre e esclarecido e, em seguida, foram entrevistadas. Em média, 20 minutos foram suficientes para concluir cada participação. Esta pesquisa faz parte de um projeto maior que conta com aprovação do Comitê de Ética em Pesquisa do Hospital Infantil Albert Sabin, Ceará.

\section{Análise dos dados}

Os dados foram tabulados e analisados através do software SPSS versão 25. Foram realizadas estatísticas descritivas (frequência, porcentagem, média e desvio padrão) para caracterização da amostra. Para testar as hipóteses da pesquisa, foram realizadas três análises de regressão múltipla hierárquica, com método enter, tendo o suporte social formal e o suporte social informal como variáveis independentes e a saúde mental, autoeficácia parental e satisfação com a vida como variáveis dependentes. Ademais, na primeira equação de cada análise foram incluídas como variáveis preditoras as variáveis sociodemográficas: número de filhos, escolaridade, renda, situação conjugal ( $0=$ solteira; 1 = casada; união estável), uso de serviços públicos ( $0=$ não; $1=\operatorname{sim}) \mathrm{e}$ privados de saúde $(0=$ não; $1=\operatorname{sim})$. As variáveis categóricas foram transformadas em variáveis dummy para utilização na análise de regressão.

\section{Resultados}

Inicialmente, para avaliar a relação entre o suporte social informal e formal e as variáveis saúde mental, satisfação com a vida e autoeficácia foram realizadas análises de correlação de Pearson. Os resultados são apresentados na Tabela 1. Quanto ao suporte formal, o acompanhamento por profissionais está relacionado com maior nível de autoeficácia. A percepção de suporte informal está relacionada com maior satisfação com a vida e saúde mental. Ao analisarmos as dimensões da ESSS, observamos que o fator satisfação com as amizades está correlacionado com a satisfação com a vida, saúde mental e autoeficácia parental, enquanto a intimidade se relaciona apenas com as duas primeiras enquanto que a satisfação com a família com a segunda. As atividades sociais apresentaram uma correlação marginalmente significativa com a saúde mental.

Em seguida, a primeira análise de regressão teve como variável critério a saúde mental ( $\mathrm{Ta}$ bela 2). O coeficiente de regressão obtido na primeira equação, apenas com variáveis sociodemográficas, foi significativo $(p<0,05)$. Os resultados apontam que a saúde mental é predita pelo número de filhos $(p<0,05)$, de modo que a mulheres que indicaram ter mais filhos relataram menor nível de saúde mental. $\mathrm{Na}$ segunda equação, na qual se acrescentam as variáveis de suporte social, o coeficiente de regressão obtido também foi significativo $(p<0,01)$. Os resultados apontam que o suporte social informal prediz significativamente a saúde mental $(p<0,05)$, ou seja, as mães que relataram maior percepção de suporte social informal apresentaram melhores níveis de saúde mental, corroborando com a hipótese 1. Por outro lado, o suporte social formal não prediz significativamente $(p=0,13)$ a saúde mental, rejeitando a hipótese 4 . A variável número de filhos apresentou o mesmo efeito da equação anterior. 
Tabela 1. Correlações de Pearson entre suporte social, saúde mental, satisfação com a vida e autoeficácia.

\begin{tabular}{lcccc}
\hline & M (DP) & Saúde mental & $\begin{array}{c}\text { Satisfação com } \\
\text { a vida }\end{array}$ & Autoeficácia \\
\hline Suporte social formal & $4,07(2,16)$ & $-0,104$ & 0,124 & $0,334^{* *}$ \\
Suporte social informal & $3,31(0,68)$ & $-0,508^{* *}$ & $0,413^{* *}$ & 0,194 \\
Satisfação com as amizades & $3,32(0,78)$ & $-0,548^{* *}$ & $0,439^{* *}$ & $0,289^{*}$ \\
Intimidade & $3,53(0,96)$ & $-0,445^{* *}$ & $0,372^{* *}$ & 0,237 \\
Satisfação com a família & $3,77(0,95)$ & $-0,280^{*}$ & 0,223 & $-0,062$ \\
Atividade sociais & $2,54(0,84)$ & $-0,225$ & 0,164 & 0,079 \\
\hline
\end{tabular}

Nota: ${ }^{\star} p<0,05 ;{ }^{* *} p<0,01$.

Fonte: Elaborado pelos autores.

Tabela 2. Análise de regressão para a variável critério saúde mental.

\begin{tabular}{|c|c|c|c|c|c|c|c|c|}
\hline & \multicolumn{3}{|c|}{ Modelo 1} & \multicolumn{5}{|c|}{ Modelo 2} \\
\hline & b & $\boldsymbol{\beta}$ & $p$ & $b$ & IC 95\% & $\boldsymbol{\beta}$ & $p$ & $\boldsymbol{\eta}_{p}^{2}$ \\
\hline Constante & 1,38 & & 0,00 & 2,35 & 1,$41 ; 3,28$ & & 0,00 & \\
\hline Situação conjugal & 0,22 & 0,13 & 0,370 & 0,27 & $-0,19 ; 0,73$ & 0,16 & 0,239 & 0,03 \\
\hline Número de filhos & 0,20 & $0,43^{*}$ & 0,006 & 0,17 & 0,$04 ; 0,30$ & $0,37^{*}$ & 0,011 & 0,15 \\
\hline Escolaridade & 0,05 & 0,14 & 0,371 & 0,07 & $-0,03 ; 0,17$ & 0,21 & 0,159 & 0,05 \\
\hline Renda & $-0,01$ & $-0,03$ & 0,857 & $-0,01$ & $-0,13 ; 0,11$ & $-0,02$ & 0,857 & 0,00 \\
\hline Saúde pública & 0,27 & 0,26 & 0,072 & 0,25 & $-0,02 ; 0,53$ & 0,25 & 0,071 & 0,08 \\
\hline Plano de saúde & 0,04 & 0,03 & 0,821 & 0,01 & $-0,29 ; 0,31$ & 0,01 & 0,936 & 0,00 \\
\hline SS informal & & & & $-0,26$ & $-0,46 ;-0,07$ & $-0,35^{*}$ & 0,010 & 0,15 \\
\hline \multirow[t]{2}{*}{ SS formal } & & & & $-0,05$ & $-0,11 ; 0,01$ & $-0,20$ & 0,126 & 0,06 \\
\hline & \multicolumn{3}{|c|}{$\begin{array}{l}\mathrm{F}(6,48)=2,44 \\
\quad p=0,041 ; \\
\mathrm{R}^{2}=0,26 ; \\
R_{\text {Ajustado }}^{2}=0,15\end{array}$} & \multicolumn{5}{|c|}{$\begin{array}{r}\mathrm{F}(8,48)=3,24, p= \\
\mathrm{R}^{2}=0,39 \\
R_{\text {Ajustado }}^{2}=0,27\end{array}$} \\
\hline
\end{tabular}

Nota: ${ }^{\star} p<0,05$.

Fonte: Elaborado pelos autores.

A segunda análise de regressão, que teve como variável critério a satisfação com a vida (Tabela 3), indicou que o coeficiente de regressão obtido na primeira equação não foi significativo $(p<0,05)$. No entanto, a variável número de filhos foi significativa $(p<0,05)$, de modo que mulheres que indicaram ter mais filhos relataram menor nível de satisfação com a vida. Na segunda equação, o coeficiente de regressão obtido foi significativo $(p<0,05)$. Os resultados apontam que o suporte social informal prediz significativamente a satisfação com a vida $(p<0,05)$, portanto, as mães que relataram maior percepção de suporte social informal apresentaram maiores níveis de satisfação com a vida, corroborando com a hipótese 2. Ademais, as variáveis número de filhos e escolaridade apresentaram resultados significativos $(p=0,049)$, embora limítrofes quanto ao ponto de corte da significância estatís- tica, indicando que as mulheres com mais filhos e com nível de escolaridade mais elevado tendem a apresentar menor satisfação com a vida.

Por fim, a última análise de regressão teve como variável critério a autoeficácia parental (Tabela 4). O coeficiente de regressão obtido na primeira equação não foi significativo, embora esteja próximo do ponto de corte para significância estatística $(p=0,06)$. Os resultados apontam que o sentimento de maior autoeficácia parental é predito pela renda familiar $(p<0,05)$, de modo que, pessoas com maior renda apresentam maior autoeficácia parental. Na segunda equação, na qual se acrescentam as variáveis de suporte social, o coeficiente de regressão obtido foi significativo $(p<0,01)$. Ademais, o suporte social formal prediz significativamente a autoeficácia parental $(p$ $<0,001$ ), ou seja, as mães que têm mais acesso ao atendimento de profissionais de saúde apresen- 
tam maiores níveis de autoeficácia parental, corroborando a hipótese 3. O suporte social informal não foi significativo. Ademais, ao acrescentar as variáveis de suporte, a variável renda familiar continuou predizendo significativamente a autoeficácia parental.

\section{Discussão}

O presente artigo teve por objetivo avaliar o papel preditivo do suporte social informal e formal na saúde mental, autoeficácia materna e satisfa- ção com a vida de mães de crianças com SZC. Os resultados observados são coerentes com os relatados em estudos com mães de crianças com outras condições crônicas ${ }^{1,13,18-20,28}$, que indicam que o suporte social prediz significativamente a saúde mental, satisfação com a vida e autoeficácia materna. Ao longo do teste de quatro hipóteses, os resultados apontam que a maior percepção de suporte social informal prediz melhores níveis de saúde mental (hipótese 1) e de satisfação com a vida (hipótese 2), corroborando com as hipóteses 1 e 2. Ademais, o acesso ao suporte social formal também apresenta um papel significativo no

Tabela 3. Análise de regressão para a variável critério Satisfação com a vida.

\begin{tabular}{|c|c|c|c|c|c|c|c|c|}
\hline & \multicolumn{3}{|c|}{ Modelo 1} & \multicolumn{5}{|c|}{ Modelo 2} \\
\hline & $b$ & $\beta$ & $p$ & $b$ & IC $95 \%$ & $\boldsymbol{\beta}$ & $p$ & $\eta^{2}$ \\
\hline Constante & 5,42 & & 0,00 & 3,38 & 1,$29 ; 5,47$ & & 0,00 & \\
\hline Situação conjugal & 0,31 & 0,09 & 0,552 & 0,12 & $-0,88 ; 1,12$ & 0,03 & 0,805 & 0,00 \\
\hline Número de filhos & $-0,36$ & $-0,35^{*}$ & 0,029 & $-0,30$ & $-0,61 ; 0$ & $-0,29^{*}$ & 0,049 & 0,09 \\
\hline Escolaridade & $-0,18$ & $-0,25$ & 0,137 & $-0,23$ & $-0,47 ; 0$ & $-0,31^{*}$ & 0,049 & 0,09 \\
\hline Renda & 0,10 & 0,11 & 0,487 & 0,08 & $-0,18 ; 0,35$ & 0,09 & 0,539 & 0,01 \\
\hline Saúde pública & $-0,45$ & $-0,20$ & 0,193 & $-0,43$ & $-1,08 ; 0,22$ & $-0,19$ & 0,191 & 0,04 \\
\hline Plano de saúde & 0,15 & 0,06 & 0,684 & 0,23 & $-0,46 ; 0,92$ & 0,10 & 0,509 & 0,01 \\
\hline SS informal & & & & 0,56 & 0,$1 ; 1,01$ & $0,33^{*}$ & 0,017 & 0,13 \\
\hline \multirow[t]{2}{*}{ SS formal } & & & & 0,12 & $-0,02 ; 0,27$ & 0,24 & 0,090 & 0,07 \\
\hline & \multicolumn{3}{|c|}{$\begin{array}{c}\mathrm{F}(6,50)=1,45, p=0,216 ; \\
\mathrm{R}^{2}=0,16 ; \\
R^{2}\end{array}$} & \multicolumn{5}{|c|}{$\begin{array}{c}\mathrm{F}(8,50)=2,27, p=0,04 \\
\mathrm{R}^{2}=0,30\end{array}$} \\
\hline
\end{tabular}

Nota: ${ }^{*} p<0,05$.

Fonte: Elaborado pelos autores.

Tabela 4. Análise de regressão para a variável critério Autoeficácia parental.

\begin{tabular}{|c|c|c|c|c|c|c|c|c|}
\hline & \multicolumn{3}{|c|}{ Modelo 1} & \multicolumn{5}{|c|}{ Modelo 2} \\
\hline & $b$ & $\beta$ & $p$ & $b$ & IC 95\% & $\beta$ & $p$ & $\eta_{p}^{2}$ \\
\hline Constante & 2,88 & & 0,00 & 2,44 & 1,$12 ; 3,76$ & & 0,00 & \\
\hline Situação conjugal & 0,01 & 0,00 & 0,979 & $-0,19$ & $-0,83 ; 0,46$ & $-0,08$ & 0,563 & 0,01 \\
\hline Número de filhos & $-0,02$ & $-0,02$ & 0,878 & 0,01 & $-0,17 ; 0,19$ & 0,02 & 0,908 & 0,00 \\
\hline Escolaridade & 0,03 & 0,07 & 0,664 & 0,02 & $-0,12 ; 0,16$ & 0,04 & 0,802 & 0,00 \\
\hline Renda & 0,27 & $0,43^{*}$ & 0,005 & 0,25 & 0,$08 ; 0,42$ & $0,40^{*}$ & 0,005 & 0,18 \\
\hline Saúde pública & $-0,17$ & $-0,12$ & 0,418 & $-0,24$ & $-0,63 ; 0,15$ & $-0,16$ & 0,225 & 0,04 \\
\hline Plano de saúde & $-0,46$ & $-0,30$ & 0,055 & $-0,36$ & $-0,78 ; 0,07$ & $-0,23$ & 0,099 & 0,07 \\
\hline SS informal & & & & 0,04 & $-0,23 ; 0,32$ & 0,04 & 0,754 & 0,00 \\
\hline \multirow[t]{2}{*}{ SS formal } & & & & 0,14 & 0,$06 ; 0,23$ & $0,43^{* *}$ & 0,002 & 0,22 \\
\hline & \multicolumn{3}{|c|}{$\begin{array}{c}\mathrm{F}(6,48)=2,23, p=0,059 \\
\mathrm{R}^{2}=0,24\end{array}$} & \multicolumn{5}{|c|}{$\begin{array}{c}\mathrm{F}(8,48)=3,46, p=0,004 \\
\mathrm{R}^{2}=0,41\end{array}$} \\
\hline
\end{tabular}

Nota: ${ }^{\star} p<0,05 ;{ }^{* *} p<0,01$. 
aumento da autoeficácia parental, corroborando com a hipótese 3 . Todavia, a hipótese 4, que afirma que o suporte social formal iria predizer a saúde mental das mães de crianças com SZC, não foi corroborada.

Em coerência com a literatura ${ }^{1,29}$, os resultados aqui observados indicam que o suporte social formal e informal se correlacionam de forma distinta com a saúde mental, satisfação com a vida e autoeficácia parental de mães de crianças com SZC, cabendo observar que esses efeitos se mantêm mesmo controlado pelas características sociodemográficas das mães, ou seja, o efeito do suporte social não pode ser reduzido as diferenças de caráter sociodemográfico ${ }^{1}$. O suporte social é um importante fator de proteção e promoção de saúde mental nos pais de crianças com SZC ${ }^{8,30}$, ajudando no desenvolvimento de estratégias de enfrentamento para as dificuldades de gerenciar a vida cotidiana de cuidados com essas crianças. Dessa forma, faz-se necessária atenção aos grupos de pais para verificar indicadores de problemas emocionais e de qualidade de vida, estruturando uma rede de apoio social para promover a saúde mental nessas famílias ${ }^{30}$. Embora diversos autores $^{8,30}$ e manuais ${ }^{31}$ apontem a necessidade de suporte social a famílias de crianças com SZC, esse é o primeiro estudo que demonstra empiricamente o efeito protetivo do suporte social.

A relação entre o suporte social informal, a saúde mental ${ }^{12,17}$ e a satisfação com a vida ${ }^{20}$ de mães de crianças com SZC é consistente com os resultados de estudos anteriores realizados com famílias com crianças com outras condições crônicas. Como dito anteriormente, o suporte informal é proveniente da rede social do indivíduo, oferecido geralmente por familiares, amigos, vizinhos ou conhecidos, provendo suporte emocional. Um dos mecanismos através dos quais o suporte informal influencia a saúde mental e a satisfação com a vida diz respeito ao senso de pertença e de companheirismo ${ }^{13}$. De acordo com Thoits ${ }^{13}$, fazer parte e contar com pessoas e grupos próximos aumenta o sentimento de pertença, de ligação entre as pessoas, assim como a percepção de companheirismo advindo do pertencimento a grupos familiares ou de amigos. O senso de pertença e o companheirismo estão relacionados com uma vivência maior de afetos positivos, que prediz maior bem-estar psicológico $^{32}$. Por outro lado, falta de pertença e companheirismo, comumente descritos como solidão, estão associados a consequências negativas para a saúde física e mental ${ }^{13,33}$.

Essa faceta do suporte social se faz central na realidade das mães de crianças com SCZ. Alguns estudos têm apontado que o isolamento social e a perda do senso de pertença são comuns em mães de crianças com desenvolvimento atípico, incluindo as mães de crianças com SZC ${ }^{34}$. Freitas et al. ${ }^{34}$, em um estudo que objetivou descrever as repercussões de cuidar de filhos com SZC a partir da perspectiva das mães, apontam que, com o ônus do cuidado focado nelas, muitas mulheres perdem sua identidade pessoal, principalmente aquelas sem uma rede de apoio. Ademais, devido à falta de tempo para realizar outras atividades, as mães acabam se retirando e se isolando das tarefas sociais e de lazer, aumentando o risco de adoecimento mental. Portanto, contar com outras pessoas para dividir a carga de cuidados, realizar atividades de lazer, ou mesmo para descarregar a carga emocional são aspectos benéficos da rede informal de suporte social e contribuem para evitar o isolamento social ${ }^{35}$.

A relação observada entre o suporte social formal e a autoeficácia materna é coerente com outros estudos na literatura ${ }^{36-38}$. Em geral, a avaliação dos pais sobre sua capacidade de realizar com eficácia as tarefas parentais têm consequências importantes para práticas parentais e para o desenvolvimento infantil ${ }^{39}$. De acordo com o modelo de Bandura ${ }^{40}$, os pais que possuem um alto senso de autoeficácia parental acreditam possuir as habilidades e qualidades necessárias para ter uma influência positiva no comportamento e desenvolvimento de seus filhos. Por outro lado, pais com baixa autoeficácia parental correm o risco de experimentar ansiedade, depressão, estresse e ter resultados mais desfavoráveis no tratamento de seus filhos. De acordo com a teoria de Bandu$\mathrm{ra}^{40}$, as crenças de autoeficácia são desenvolvidas e moldadas por experiências de sucesso e fracasso, dificuldade percebida das tarefas, interações interpessoais com outros e excitação fisiológica e psicológica aversiva, como a ansiedade.

De acordo com Shorey, Chan, Chong e $\mathrm{He}^{36}$ as evidências de pesquisas apoiam que a experiência vicária e os componentes de persuasão verbal da teoria da autoeficácia de Bandura estão ligados ao apoio social, e que esse tem uma forte influência na autoeficácia ${ }^{38}$. Observa-se que as mães com melhor suporte social tendem a se perceber portadora de maior autoeficácia materna ${ }^{36,38,41}$. Portanto, esses achados apontam para a necessidade de os profissionais das políticas públicas de assistência à saúde planejarem intervenções apropriadas para melhorar a autoeficácia materna e, consequentemente, sua saúde mental. Embora se observe na literatura uma relação entre suporte social formal e autoeficácia materna, esperava-se encontrar uma relação positiva também com a saúde mental, já que a autoeficácia materna tem 
sido comumente empregada como um fator mediador/moderador entre variáveis psicossociais (a exemplo do suporte social) e a saúde mental ${ }^{39}$. No entanto, é possível que não se tenha observado uma correlação significativa entre o suporte formal e a saúde mental em função da estratégia de mensuração do suporte formal utilizada no presente estudo. Estudos futuros poderão adotar medidas mais amplas de suporte social formal que avaliem os aspectos informativos, instrumentais e emocionais dessa fonte de suporte social, possibilitando uma avaliação mais acurada da sua relação com dimensões da saúde mental.

Paralelamente ao suporte social formal e informal, observamos que algumas variáveis sociodemográficas, destacando-se a renda econômica e o número de filhos, explicaram significativamente as variáveis dependentes utilizadas nesse estudo. $\mathrm{O}$ fator econômico está associado a epidemiologia da SZC, já que ela atingiu de forma predominante a população de baixa renda e que habitava em localidades com pouca ou nenhuma infraestrutura sanitária ${ }^{3}$. Ademais, a renda pode constituir uma limitação no acesso aos meios de suporte social formal. Embora o modelo de saúde pública e universal do SUS preconize a integralidade do acesso à saúde, estudos anteriores têm apontado os desafios que as mães de crianças com SZC enfrentam para ter acesso aos serviços públicos de saúde ${ }^{30,34}$. As condições de desigualdade social e de pobreza tendem a ser exacerbadas após o nascimento de um filho com SZC, pois muitas mães relatam a perda ou o abandono do emprego devido às necessidades de cuidados e a rotina de tratamentos demandadas pelas crianças com SZC. Como apontado anteriormente, $81 \%$ das mães entrevistadas estavam desempregadas. O percentual de mães empregadas era de 13,4\%, ante $67,6 \%$ que indicaram possuir um trabalho formal antes de ter o filho com SZC. De acordo com Freitas et al. ${ }^{34}$ a luta para sobreviver, prover tratamento e atender às necessidades da família é recorrente nas narrativas de muitas mães. Dessa forma, muitas famílias encontram dificuldades, por exemplo, para o descolamento até os serviços públicos de saúde, sobretudo para aquelas que residem em municípios distantes dos grandes centros urbanos, uma vez que os tratamentos são majoritariamente oferecidos nesses centros. Esses fatores podem, em última análise, reduzir a percepção de autoeficácia para lidar com as demandas dos filhos com SZC, já que essa é mais fortemente explicada pelo suporte social formal.

Observou-se que as mães que relataram ter mais filhos apresentaram menor saúde mental e menor satisfação com a vida. Isso reflete, em grande parte, os papéis sociais tradicionalmente desempenhados pelas mulheres, que envolvem o cuidado majoritário dos membros da família. De fato, as mulheres continuam a assumir a responsabilidade primária pela assistência aos filhos e por outros serviços domésticos ${ }^{3}$. Pesquisas existentes têm indicado que, comparativamente com as mães, os pais de crianças com desenvolvimento atípico são menos envolvidos nos cuidados diários com as crianças e, consequentemente, apresentam melhores níveis de saúde mental ${ }^{10}$. Portanto, as cargas mental e física ${ }^{42}$ que recaem mais fortemente sobre as mães acabam por reduzir sua saúde mental e satisfação com a vida.

Ademais, observou-se um efeito da escolaridade na satisfação com a vida, indicando que mães com maior escolaridade reportaram menor satisfação com a vida. No entanto, esse resultado deve ser analisado com cautela. A variabilidade em termos de escolaridade foi baixa nessa amostra: apenas 14,7\% das mães indicaram possuir Ensino Superior completo ou incompleto, evidenciando a maior prevalência da SZC entre pessoas de classe de baixa renda e escolaridade; o valor de $p=0,049$ ficou ligeiramente abaixo do ponto de corte da significância estatística $(p$ $=0,05)$, levantando receios de que o efeito seja um falso-positivo ou que tenha pouca significância prática ${ }^{43}$, nomeadamente por não ser corroborado por outros estudos na literatura, que têm indicado que a satisfação com a vida é invariante entre os níveis educacionais ${ }^{44}$. Estudos futuros com maior variabilidade amostral em relação à escolaridade poderão apresentar resultados mais confiáveis sobre o efeito dessa variável na satisfação com a vida.

De modo geral, embora os resultados obtidos sejam consistentes teoricamente e coerentes com os achados de outros estudos, o delineamento correlacional aqui adotado apresenta uma limitação, já que não nos permite afirmar a direção da relação entre as variáveis estudadas. Estudos futuros, com método longitudinal, poderão esclarecer mais adequadamente o papel do suporte social na saúde mental de mães de crianças com SZC. Ademais, as mães que participaram desse estudo haviam procurado serviços de saúde especializados para os filhos, onde foram entrevistadas, o que pode estar relacionado a um maior apoio formal. Entrevistar mães que não se encontram nesses serviços pode trazer um panorama diferente do aqui apresentado, já que essas possivelmente apresentam níveis menores de suporte social formal. 
Em síntese, o presente estudo apresenta uma contribuição inédita para a compreensão do papel do suporte social na saúde mental de mães de crianças com SZC. O suporte social formal e informal apresentaram padrões de predição distintos com a saúde mental, satisfação com a vida e autoeficácia materna, apontando para a necessidade dessas duas fontes de suporte serem vistas de forma complementar.

\section{Referências}

1. Gouin JP, Estrela C, Desmarais K, Barker ET. The Impact of Formal and Informal Support on Health in the Context of Caregiving Stress. Fam Relat 2016 65(1):191-206.

2. Guillamón N, Nieto R, Pousada M, Redolar D, Muñoz E, Hernández E, Boixadós M, Gómez-Zúñiga B. Quality of life and mental health among parents of children with cerebral palsy: The influence of self-efficacy and coping strategies. J Clin Nurs 2013; 22(1112):1579-1590.

3. Souza LEC, Lima TJS, Ribeiro EM, Pessoa ALS, Figueiredo TC, Lima LBP. Mental Health of Parents of Children with Congenital Zika Virus Syndrome in Brazil. J Child Fam Stud 2018; 27(4):1207-1215.

4. Oliveira SJGS, Melo ES, Reinheimer DM, Gurgel RQ, Santos VS, Martins-Filho PRS. Anxiety, depression, and quality of life in mothers of newborns with microcephaly and presumed congenital Zika virus infection. Arch Womens Ment Health [Internet]. Arch Womens Ment Health 2016; 19(6):1149-1151.

5. Costa PRLA. Percepção de qualidade de vida de mães de crianças portadoras de síndrome congênita associada ao zika vírus [dissertação]. São Luís: Universidade Federal do Maranhão; 2018.

6. Menezes ASS, Alves MJS, Gomes TP, Pereira JA. Microcefalia relacionada ao vírus Zika e dinâmica familiar: perspectiva da mãe. Av en Enfermería 2019; 37(1):38-46.

7. Bromley J, Hare DJ, Davison K, Emerson E. Mothers supporting children with autistic spectrum disorders: Social support, mental health status and satisfaction with services. Autism 2004; 8(4):409-423.

8. Bailey DB, Ventura LO. The Likely Impact of Congenital Zika Syndrome on Families: Considerations for Family Supports and Services. Pediatrics 2018; 141(Supl. 2):S180-S187.

\section{Colaboradores}

TJS Lima colaborou na elaboração do delineamento da pesquisa, análise dos dados e escrita do manuscrito. LEC Souza colaborou na elaboração do delineamento da pesquisa, coleta dos dados, discussão dos resultados e revisão final do manuscrito.

9. Prakash V, Patel AM, Hariohm K, Palisano RJ. Higher Levels of Caregiver Strain Perceived by Indian Mothers of Children and Young Adults with Cerebral Palsy Who have Limited Self-Mobility. Phys Occup Ther Pediatr 2017;37(1):64-73.

10. Factor RS. Autism spectrum disorder traits and parental stress: The moderating role of parental self-efficacy. 2016;1-93. [cited 2019 Mai 3]. Available from: https:/vtechworks.lib.vt.edu/bitstream/ handle/10919/77505/etd-11172016-101249_Factor RS_T_2016.pdf?sequence $=1$ \&isAllowed $=y$

11. Dabrowska A, Pisula E. Parenting stress and coping styles in mothers and fathers of pre-school children with autism and Down syndrome. J Intellect Disabil Res 2010; 54(3):266-280

12. Hsiao YJ. Pathways to mental health-related quality of life for parents of children with autism spectrum disorder: Roles of parental stress, children's performance, medical support, and neighbor support. Res Autism Spectr Disord. Elsevier Ltd; 2016;23:122-130.

13. Thoits PA. Mechanisms linking social ties and support to physical and mental health. J Health Soc Behav 2011; 52(2):145-161.

14. Brofenbrenner U,Morris P.The Bioecological Model Of Human Development. Handb Child Psychol [Internet]. Abingdon, Oxon ; New York, NY : Routledge, 2017. Series: Introducing early years thinkers: Routledge; 2007. p. 13-27. [cited 2019 Maio 3]. Available from: https:// www.taylorfrancis.com/books/9781317294313/chapters/10.4324/9781315646206-2

15. Pickard KE, Ingersoll BR. Quality versus quantity: The role of socioeconomic status on parent-reported service knowledge, service use, unmet service needs, and barriers to service use. Autism 2016; 20(1):106115.

16. Searing BMJ, Graham F, Grainger R. Support Needs of Families Living with Children with Autism Spectrum Disorder. J Autism Dev Disord 2015; 45(11):36933702 . 
17. Gariépy G, Honkaniemi H, Quesnel-Vallée A. Social support and protection from depression: Systematic review of current findings in western countries. $\mathrm{Br} \mathrm{J}$ Psychiatry 2016; 209(4):284-293.

18. Zaidman-Zait A, Mirenda P, Duku E, Vaillancourt T, Smith IM, Szatmari P, Bryson S, Fombonne E, Volden J, Waddell C, Zwaigenbaum L, Georgiades S, Bennett T, Elsabaggh M, Thompson A. Impact of personal and social resources on parenting stress in mothers of children with autism spectrum disorder. Autism 2017; 21(2):155-166.

19. Singh P, Ghosh S, Nandi S. Subjective Burden and Depression in Mothers of Children with Autism Spectrum Disorder in India: Moderating Effect of Social Support. J Autism Dev Disord 2017; 47(10):3097-3111.

20. Lu M, Yang G, Skora E, Wang G, Cai Y, Sun Q, Li W. Self-esteem, social support, and life satisfaction in Chinese parents of children with autism spectrum disorder. Res Autism Spectr Disord 2015; 17:70-77.

21. McIntyre LL, Brown M. Examining the utilisation and usefulness of social support for mothers with young children with autism spectrum disorder. $J$ Intellect Dev Disabil 2018; 43(1):93-101.

22. Warren JS, Brown CR, Layne CM, Nelson PL. Parenting self-efficacy as a predictor of child psychotherapy outcomes in usual care: A multi-dimensional approach. Psychother Res 2011; 21(1):112-123.

23. Meirsschaut M, Roeyers H, Warreyn P. Parenting in families with a child with autism spectrum disorder and a typically developing child: Mothers' experiences and cognitions. Res Autism Spectr Disord 2010; 4(4):661-669.

24. Marôco JP, Campos JADB, Vinagre MG, Pais-Ribeiro JL. Adaptação Transcultural Brasil-Portugal da Escala de Satisfação com o Suporte Social para Estudantes do Ensino Superior. Psicol Reflexão e Crítica 2014; 27(2):247-256.

25. Gouveia VV, Lima TJS, Gouveia RSV, Freires LA, Barbosa LHGM. General Health Questionnaire (GHQ12): The effect of negative items in its factorial structure. Cad Saude Publica 2012; 28(2):375-384.

26. Lawton MP, Moss M, Hoffman C, Perkinson M. Two Transitions in Daughters ' Caregiving Careers. Gerontol 2000; 40(4):437-448.

27. Diener E, Emmons RA, Larsen RJ, Griffin S. The Satisfaction With Life Scale. J Pers Assess 1985; 49(1):71-75.

28. Shiba K, Kondo N, Kondo K. Informal and formal social support and caregiver burden: the AGES caregiver survey. J Epidemiol 2016; 26(12):622-628.

29. White N, Hastings RP. Social and professional support for parents of adolescents with severe intellectual disabilities. J Appl Res Intellect Disabil 2004; 17(3):181190.

30. Brunoni D, Blascovi-Asis SM, Osório AAC, Seabra AG, Amato CAH, Teixeira MC, Roch MM, Carreiro LRR. Microcefalia e outras manifestações relacionadas ao vírus Zika: impacto nas crianças, nas famílias e nas equipes de saúde. Cien Saude Colet 2016; 21:32973302.

31. Brasil. Ministério da Saúde (MS). Apoio psicossocial a mulheres gestantes, famílias e cuidadores de crianças com síndrome congênita por vírus zika e outras deficiências: guia de práticas para profissionais e equipe de saúde. Brasília: MS; 2017.
32. Diener E, Chan MY. Happy People Live Longer: Subjective Well-Being Contributes to Health and Longevity. Appl Psychol Heal Well-Being 2011; 3(1):1-43.

33. Vasileiou K, Barnett J, Barreto M, Vines J, Atkinson M, Lawson S, Wilson M. Experiences of loneliness associated with being an informal caregiver: A qualitative investigation. Front Psychol 2017; 8(Apr):1-11.

34. Freitas PSS, Soares GB, Mocelin HJS, Lamonato CCXL, Sales CMM, Linde-Arias AR, Bussinger ECA, Maciel ELN. How do mothers feel? Life with children with congenital Zika syndrome. Int J Gynecol Obstetrics 2020; 148:20-28.

35. Ruiz-Robledillo N, Andrés-García S, Pérez-Blasco J, González-Bono E, Moya-Albiol L. Highly resilient coping entails better perceived health, high social support and low morning cortisol levels in parents of children with autism spectrum disorder. Res Dev Disabil 2014; 35(3):686-695.

36. Shorey S, Chan SWC, Chong YS, He HG. Predictors of Maternal Parental Self-Efficacy Among Primiparas in the Early Postnatal Period. West J Nurs Res 2015; 37(12):1604-1622.

37. Shorey S, Chan SWC, Chong YS, He HG. A randomized controlled trial of the effectiveness of a postnatal psychoeducation programme on self-efficacy, social support and postnatal depression among primiparas. J Adv Nurs 2015; 71(6):1260-1273.

38. Liyana Amin NA, Tam WWS, Shorey S. Enhancing first-time parents' self-efficacy: A systematic review and meta-analysis of universal parent education interventions' efficacy. Int J Nurs Stud 2018; 82(March):149-162.

39. Smart LK. Parenting Self-Efficacy in Parents of Children with Autism Spectrum Disorders. ProQuest Diss Theses 2016; 121.

40. Bandura A. Social cognitive theory: An agentic perspective. Annu Rev Psychol 2001; 52(1):1-26.

41. Leahy-Warren P, Mccarthy G, Corcoran P. First-time mothers: Social support, maternal parental self-efficacy and postnatal depression. J Clin Nurs 2012;21(34):388-397.

42. Faircloth C. When equal partners become unequal parents: couple relationships and intensive parenting culture. Fam Relatsh Soc 2020; Mar.

43. Checa I, Perales J, Espejo B. Measurement invariance of the Satisfaction with Life Scale by gender, age, marital status and educational level. Quality of Life Research 2019; 28(4):963-968.

44. Ioannidis JP. The proposal to lower P value thresholds to. 005. Jama 2018; 319(14):1429-1430.

Artigo apresentado em 23/05/2019

Aprovado em 08/06/2020

Versão final apresentada em 10/06/2020

Editores-chefes: Romeu Gomes, Antônio Augusto Moura da Silva 